\title{
Briefe An Die Herausgeber - Letters To The Editors
}

L. Heilmann, E. Lorch, B. Hojnacki, H. Müntefering, H. Förster: Die Speicherung von zwei unterschiedlichen Hydroxyäthylstärke-Präparaten in der Plazenta nach Hämodilution bei Patientinnen mit fetaler Mangelent-wïcklung oder Schwangerschaftshochdruck. Infusionstherapie 1991; 18:236-243.

Zur oben genannten Arbeit möchte ich einige kritische Be-merkungen machen. Die Autoren schreiben in der Zusammenfassung von «Dif-ferenzen der physikochemikalischen Charakteristika». Dabei bleiben sie aber die Erläuterung dieser Differenzen schuldig. Der Grundstoff ist in beiden Präparaten gleich, obwohl in den Wissenschaftlichen Broschüren unterschiedliche Ausdrücke gewählt werden. Es bestehen tatsächlich Unterschiede. Sie be-ruhen aber auf einem unterschiedlichen Mw/Mn-Verhältnis, welches mit 1,81 beim HES B1 besser ausfällt als mit 3,49 beim HES A2 [1]. Da bei der Präparation B weniger großmolekula-re Bestandteile vorhanden sind, müßte die Speicherung gerin-ger ausfallen. Die höhere Speicherung in der Nabelschnur läßt sich aber durch den Versuchsaufbau erklären. Da das Intervall zwischen Infusionsende und Geburt (ich nehme an, daß dies in der 7. Reihe der Tab. 2 gemeint ist) bei der Gruppe A 7,1 Tage und bei der Gruppe B 0,3 Tage beträgt, dürften die Ergebnisse dadurch erklärt sein.

Der Faktor VחIR:Ag (von-Willebrand-Faktor) dürfte bei diesem Patientengut kein guter Indikator für eine Störung des Gerinnungssystems sein. Er ist bei Patientinnen mit Schwangerschaftshochdruck erhöht [2]. Daher kann der Abfall auf 115\% (!) der Norm auch als bessere Therapie ausgelegt werden.

Die größere Störung des Gerinnungssystems muß auch in Frage gestellt werden. Zum einen wird nicht dargelegt, ob die Veränderungen von PTT und Faktor VIIR:Ag zwischen den beiden HESGruppen signifikant sind. Zum anderen spielt der Zeitpunkt der zweiten Probennahme eine entscheidende Rol-le. Wurden diese Proben unmittelbar vor der Geburt oder kur-ze Zeit nach der Geburt abgenommen? Eine Probengewin-nung bei einer bestehenden atonischen (!) Blutung wird eine leichte Störung der Blutgerinnung aufzeigen. Die signifikante Verlängerung der PTT auf 38,9 Sekunden ist mit Sicherheit nicht der Grund für die postpartalen Blutungen.

\section{$>: 214$}

Literatur

Nitsch E: Chemie der Hydroxyethylstärke (HES). Beitr Anaesth Intensivmed 1988;26:15-26. Brenner B, Zwang E, Bronshtein M, Seligsohn U: Von Willebrand factor multimer patterns in pregnancy-induced hypertension. Thromb Haemost 1989;62:715-717.

H.-B. Simon, Aachen

Stellungnahme zum Kommentar von H.-B. Simon

Die kritische Stellungnahme von H.-B. Simon unterstreicht die Aktualität unserer Arbeit hinsichtlich des Einsatzes von Hydroxyäthylstärke-Präparaten. Daß bei HES B ein schmäleres Mw/Mn-Verhältnis vorliegt als bei HES A, war uns bekannt und könnte sicher ebenfalls eine Erklärungsmöglichkeit sein. Unsere histologischen Untersuchungen der Plazenta konnten leider nicht durch qualitative Messungen der HES-Konzentra-tion im Mutterkuchen belegt werden. 
Darüber hinaus ist es beim klinischen Einsatz der Hydroxyäthylstärke äußerst schwierig, vergleichbare Intervalle zu erhalten. Der Faktor VIIR:Ag ist sowohl in der Schwangerschaft als auch bei der Gestose erhöht. Ein Abfall des Faktors VIIIR: Ag ist durchaus erwünscht, aber ohne die Hämostase-Balance zu stören. Der von uns beschriebene stärkere Abfall des v.- WillebrandFaktors (Blutentnahme zum Zeitpunkt der Geburt und vor der Atonie) bei HES B ist bei höher substituier-ten Stärken beschrieben worden und geht mit einer vermehr-ten Blutungsneigung einher $[1,2]$.

Unsere Arbeit sollte vor allem dem Kliniker Anhaltspunkte zum differenzierten Einsatz der Hydroxyäthylstärke in der Geburtshilfe geben.

\section{Literatur}

Strauss RC, Stansfield C, Henrichsen RA, Vilhauer PJ: Pentostarch may cause fewer effects on coagulation than hetstarch. Transfus 1988;28:257-260.

Strauss RC, Stemp DC, Henrichsen RA: Hydroxyethyl starch accentuates on Willebrand $1 / 8$ disease. Transfus 1985;25:235-237.

L. Heilmann, Rüsselsheim H. Förster, Frankfurt

Haemofusin [Kabi-Pfrimmer, Erlangen; Laevosan, Linz (A)]. 2 HAES-steril 10\% (Fresenius AG, Oberursel).

(C) 1992 S. Karger GmbH, Freiburg 